\title{
Power System Dynamic State Estimation: Motivations, Definitions, Methodologies and Future Work
}

\author{
IEEE Task Force on Power System Dynamic State and Parameter Estimation \\ Junbo Zhao (TF Chair), Member, IEEE, Antonio Gómez-Expósito, Fellow, IEEE, Marcos Netto, Student \\ Member, IEEE, Lamine Mili, Life Fellow, IEEE, Ali Abur, Fellow, IEEE, Vladimir Terzija, Fellow, IEEE, \\ Innocent Kamwa, Fellow, IEEE, Bikash Pal, Fellow, IEEE, Abhinav Kumar Singh, Member, IEEE, Junjian Qi, \\ Senior Member, IEEE, Zhenyu Huang, Fellow, IEEE, A. P. Sakis Meliopoulos, Fellow, IEEE
}

\begin{abstract}
This paper summarizes the technical activities of the Task Force on Power System Dynamic State and Parameter Estimation. This Task Force was established by the IEEE Working Group on State Estimation Algorithms to investigate the added benefits of dynamic state and parameter estimation for the enhancement of the reliability, security, and resilience of electric power systems. The motivations and engineering values of dynamic state estimation (DSE) are discussed in detail. Then, a set of potential applications that will rely on DSE is presented and discussed. Furthermore, a unified framework is proposed to clarify the important concepts related to DSE, forecasting-aided state estimation, tracking state estimation and static state estimation. An overview of the current progress in DSE and dynamic parameter estimation is provided. The paper also provides future research needs and directions for the power engineering community.
\end{abstract}

Index Terms-Dynamic state estimation, Kalman filtering, synchrophasor measurements, static state estimation, tracking state estimation, forecasting-aided state estimation, parameter estimation, robust estimation, bad data, power system protection, power system dynamics, power system stability and control.

\section{INTRODUCTION}

$\mathbf{P}$ OWER systems are planned to be operated and controlled in a hierarchical manner [1]. The controls are particularly designed to deal with a variety of dynamic phenomena at multiple time scales [2]. For example, synchronous generator's automatic voltage control is based on locally available measurements only. However, their voltage set points can be

Junbo Zhao, Marcos Netto and Lamine Mili are with the Bradley Department of Electrical and Computer Engineering, Virginia Polytechnic Institute and State University, Falls Church, VA 22043, USA (e-mail: zjunbo@vt.edu)

Antonio Gómez-Expósito is with the Department of Electrical Engineering, University of Seville, Seville 41092, Spain (e-mail: age@us.es).

Ali Abur is with the Department of Electrical and Computer Engineering, Northeastern University, Boston, MA, USA (e-mail: abur@ece.neu.edu).

Vladimir Terzija is with the School of Electrical and Electronic Engineering, The University of Manchester (email: vladimir.terzija@manchester.ac.uk).

Innocent Kamwa is with Hydro-Quebec/IREQ, Power Systems and Mathematics, Varennes, Canada (e-mail: kamwa.innocent@ireq.ca).

Bikash Pal is with the Department of Electrical and Electronic Engineering, Imperial College London SW7 2BT, U.K. (e-mail: b.pal@imperial.ac.uk).

Abhinav Kumar Singh is with the School of Electrical and Electronic Engineering, University of Southampton, UK (e-mail: aks@ecs.soton.ac.uk).

Junjian Qi is with the Department of Electrical and Computer Engineering, University of Central Florida, Orlando, FL 32816 USA (e-mail: Junjian.Qi@ucf.edu).

Zhenyu Huang is with the Pacific Northwest National Laboratory, Richland, WA 99352, USA (email: zhenyu.huang@pnnl.gov).

A. P. S. Meliopoulos is with the School of Electrical and Computer Engineering, Georgia Institute of Technology, Atlanta, GA 30339 USA (email: sakis.m@gatech.edu). modified when the command from the centralized control center is received. Hence, there exists a hierarchical decentralized closed-loop control that responds to system variations and to set point changes. The centralized open-loop control is triggered by the operator after a decision-making process. In accordance with this philosophy of design, the majority of the monitoring and control applications at the control center rely on the steady-state model of the system. However, in reality, power systems never operate in steady-state condition as there are stochastic variations in demand and generation. The situation is further aggravated by the large-scale integration of distributed energy resources (DERs) on the generation side, and complex loads and new demand-response technologies on the demand side, such as electric vehicles and internet of things devices. Such a shift has given rise to larger uncertainties of the system dynamic characteristics. Consequently, the steadystate assumption becomes questionable, and static state estimation (SSE) methods unable to capture these dynamics in an operational environment. As a result, SSE methods [3], [4] used in today's energy management systems (EMS) should be reassessed and enhanced with new monitoring tools, such as dynamic state estimation (DSE). DSE is capable of accurately capturing the dynamics of the system states, and will play an important role in power system control and protection [5]-[9], especially with the increasing complexity resulting from the uncertainties by the new technologies being deployed in the generation and demand sides.

With the widespread deployment of phasor measurement units (PMUs) and advanced communication infrastructure in power systems [10], [11], the development of a fast and robust DSE tool has become possible. Despite the extensive research work on this topic, there is still a lack of common agreement on what constitutes DSE, how it should be implemented and effectively utilized in today's power system EMS. In particular, the motivations for DSE have not been thoroughly discussed and agreed upon. As a result, its engineering value is currently not fully clear to the power industry and software developers. To this end, the IEEE Working Group on State Estimation Algorithms has established a 3-year long task force on Power System Dynamic State and Parameter Estimation with the objective of creating a reference document describing the motivations, concepts, implementation and utilization of DSE for more resilient and reliable operation of future power systems. 
In this paper, DSE is presented in a unified framework, which uses commonly accepted notations and formulations in power system dynamics and control literature with the aim of establishing a firm baseline for future research and development efforts. The similarities and differences between DSE and other existing estimation methods, including forecastingaided state estimation (FASE), tracking state estimation (TSE) and SSE, are clarified. Potential applications of DSE are identified and discussed, in an effort to justify its significance for enhanced monitoring, protection and control.

The remainder of the paper is organized as follows: Section II outlines the motivations for DSE; a new unified state estimation framework that includes various existing state estimation methods is proposed in Section III; Section IV provides a thorough review of various dynamic state and parameter estimation methods followed by some discussions; Section V concludes the paper and provides future research needs and directions for the power engineering community.

\section{Motivations For Dynamic State Estimation}

With the increasing penetration of DERs, responsive loads, and microgrids, the power system has been subject to different types of dynamics. For example, the stochastic and intermittent characteristics of DERs increase the probability of sudden changes in the bus voltage phasors in a short time-frame [12]. These changes are mainly driven by the changes in active and/or reactive power injections, which further cause changes in the generator's state variables, such as rotor speeds and rotor angles. The majority of today's monitoring and control tools at the control center EMS are based on steady-state power system models, which cannot capture the system dynamics [13]. This limitation is primarily due to the fact that the EMS functions rely on the SCADA systems, which have slow scan rates and no timestamps. Therefore, the state estimates are updated every few seconds to minutes, and most of the control schemes associated with the generators or the FACTS devices are based on locally available information and measurements.

With the increased number of installed PMUs, the development of DSE for power system monitoring, control, and protection becomes possible. The benefits of using DSE include but are not limited to:

- Improved oscillations monitoring: the estimated dynamic state variables can be used to carry out modal analysis [14], and the identified modes can then be utilized to adaptively tune power system stabilizers (PSS), thereby achieving better damping of inter-area modes of oscillation and improving system stability. Recall that the effectiveness of conventional PSS using local measurements may be limited by the observability of the modes in the signal. Using state estimates of entire regions as opposed to only local measurements will increase the stabilizer's response to inter-area modes if the generator has significant influence on such modes. Note that there are several ways for monitoring the entire regions/systems via DSE, namely the hierarchical and distributed DSE and the centralized DSE using high-performance computing technique [15], [16] or reduced order model of power system [17]. Hierarchical and distributed DSE methods are first implemented locally to monitor small areas, and their results are submitted to the control center for further processing. This is the widely used strategy in the current literature. The high-performance computing techniquebased DSE for large-scale systems is in its infancy and merits further research;

- Enhanced hierarchical decentralized control [18]-[20]: the availability of local and wide-area dynamic states obtained from DSE enables the design of effective local and wide-area controls; for instance, the estimated rotor speed and other states can be used as input signals to control excitation systems of synchronous machines [19], [20] or of FACTS devices [18] so as to damp out oscillations. The implementation can be in either fully decentralized or hierarchically decentralized manner;

- Improved dependability and reliability of protection systems [6], [21]-[23]: by testing the consistency between the PMU measurements and the dynamical model of the protection zone for which the parameters are identified by DSE, both internal and external faults can be effectively detected without any a priori protection relay settings, yielding more reliable protection systems compared with the traditional coordinated settings-based schemes; the estimated online dynamic states can be utilized to initiate effective generator out-of-step protections [9], [23] and transient stability monitoring based on the extended equal-area criterion or the energy function approach [23]; furthermore, fast state estimation is a prerequisite for the implementation of system integrity protection schemes that can prevent blackouts;

- Enhanced reliability of the system models utilized for dynamic security assessment (DSA) [24]: DSA requires the availability of accurate models of the generators and their associated controllers, of the composite loads and of the special protection schemes, to name a few. By developing DSE, both the static and dynamic models can be validated [25], and if incorrect parameter values are identified, they can be included as additional state variables in DSE for parameter estimation and calibration, yielding improved system models [26] and more reliable DSA;

- Other applications: improved synchrophasor data quality and cyber security leveraging the model information, such as filtering out measurement error that is modeled as Gaussian or non-Gaussian distribution [27], [28], detecting bad and delayed measurements, or recovering missing data [29]; enhanced synchronous generators coherency identification and dynamic model reduction [30] using the estimated dynamic states and parameters; enhanced bus frequency and center of inertia estimation [31], [32] and detection of failures in controllers, such as excitation systems, PSS, etc., [33], [34].

\section{UNIFIED STATE ESTIMATION FRAMEWORK}

This section develops a unified framework for various power system state estimation, namely DSE, FASE, TSE and SSE. Specifically, the system operating conditions and the associated mathematical models are first revisited. Then, the resulting state transition expressions are identified, and 
subsequently customized for transient and quasi-steady state operating conditions.

\section{A. Quasi-Steady State versus Transient Operating Conditions}

The states of a system are essentially in any of two mutually exclusive operating conditions, quasi-steady and transient [35].

Transient operating condition: arises when a sudden disturbance takes place in the system. In this context, only electromechanical transients are considered and the associated governing equations are those customarily adopted for transient stability analysis, given by

$$
\begin{gathered}
\dot{\boldsymbol{x}}(t)=\boldsymbol{f}(\boldsymbol{x}(t), \boldsymbol{y}(t), \boldsymbol{u}(t), \boldsymbol{p}), \quad \mathbf{0}=\boldsymbol{g}(\boldsymbol{x}(t), \boldsymbol{y}(t), \boldsymbol{u}(t), \boldsymbol{p}), \\
\boldsymbol{x}_{i}^{\text {min }} \leq \boldsymbol{x}_{i} \leq \boldsymbol{x}_{i}^{\text {max }}, i \in \Xi \quad \boldsymbol{u}_{j}^{\text {min }} \leq \boldsymbol{u}_{j} \leq \boldsymbol{u}_{j}^{\text {max }}, j \in \Omega \\
\boldsymbol{p}_{l}^{\text {min }} \leq \boldsymbol{p}_{l} \leq \boldsymbol{p}_{l}^{\text {max }}, l \in \Gamma
\end{gathered}
$$

where $\boldsymbol{x} \in \mathbb{R}^{n}$ represents the system state vector, such as the internal states of a machine or a dynamic load, etc.; $\boldsymbol{y} \in \mathbb{R}^{m}$ represents the algebraic state vector, such as voltage and current phasors; note that the algebraic equations include those that are associated with the power flows and generators' stator [35]; $\boldsymbol{u}$ is the system input vector; $\boldsymbol{p}$ represents the model parameters; and $\boldsymbol{f}$ and $\boldsymbol{g}$ are nonlinear functions; due to physical limitations, controller design, some dynamic state variables, control inputs and model parameters are bounded by their upper and lower limits, which are represented by the sets $\Xi, \Omega$ and $\Gamma$, respectively [35].

Quasi-steady state operating condition: refers to the situation in which the system operating point changes exclusively due to slow and smooth load/renewable generation changes. In this scenario, the generators and other controllers are able to almost instantly absorb these slow changes, yielding negligible changes of the dynamic states $\boldsymbol{x}(t)$, i.e., $\dot{\boldsymbol{x}}(t) \approx \mathbf{0}$. As a result, the system is mathematically characterized by:

$$
\begin{aligned}
& \mathbf{0} \approx \boldsymbol{f}(\boldsymbol{x}(t), \boldsymbol{y}(t), \boldsymbol{u}(t), \boldsymbol{p}), \\
& \mathbf{0}=\boldsymbol{g}(\boldsymbol{x}(t), \boldsymbol{y}(t), \boldsymbol{u}(t), \boldsymbol{p}),
\end{aligned}
$$

subject to equality and inequality constraints, where slowly varying voltage phasors, i.e., algebraic state variables, are of interest. Since system inputs are usually not perfectly known and the parameters are always inaccurate to a certain extent, state estimators capable of processing measurement snapshots are developed, including FASE, TSE and SSE. It is worth emphasizing that, at the distribution level, individual loads may change abruptly enough for the distributed generators or local controllers to be subject to disturbances, requiring the dynamic model (1) to be resorted to.

In practice, the continuous-time models for both transient and quasi-steady state operating conditions are transformed into their discrete-time state space forms through some time discretization technique [36]. Then (1) can be written as

$$
\begin{aligned}
\boldsymbol{x}_{k} & =\boldsymbol{f}\left(\boldsymbol{x}_{k-1}, \boldsymbol{y}_{k-1}, \boldsymbol{u}_{k}, \boldsymbol{p}\right)+\boldsymbol{w}_{k}, \\
\mathbf{0} & =\boldsymbol{g}\left(\boldsymbol{x}_{k}, \boldsymbol{y}_{k}, \boldsymbol{u}_{k}, \boldsymbol{p}\right)+\boldsymbol{e}_{k},
\end{aligned}
$$

where $\boldsymbol{w}_{k}$ and $\boldsymbol{e}_{k}$ are error terms that include time discretization and model approximation errors. By treating the equality constraints (4) as pseudo-measurements and processing them together with the incoming measurements, a more general state space model for state estimation is

$$
\begin{aligned}
\boldsymbol{x}_{k} & =\boldsymbol{f}\left(\boldsymbol{x}_{k-1}, \boldsymbol{y}_{k-1}, \boldsymbol{u}_{k}, \boldsymbol{p}\right)+\boldsymbol{w}_{k}, \\
\boldsymbol{z}_{k} & =\boldsymbol{h}\left(\boldsymbol{x}_{k}, \boldsymbol{u}_{k}, \boldsymbol{p}\right)+\boldsymbol{v}_{k},
\end{aligned}
$$

subject to the constraints defined before, where $z_{k}$ is the measurement vector, including pseudo-measurements, measured algebraic variables, real and reactive power injections and flows, current phasors, etc. [10]; $\boldsymbol{h}$ is the nonlinear measurement function; $\boldsymbol{v}_{k}$ is measurement error. The $\boldsymbol{w}_{k}$ and $\boldsymbol{v}_{k}$ are usually assumed to be normally distributed with zero mean and covariance matrices, $\boldsymbol{Q}_{k}$ and $\boldsymbol{R}_{k}$, respectively. Note that they are the superposition of different sources of noise/errors (e.g. from sensors, communication channels, or models) and may not follow a Gaussian distribution in practice [37].

To perform state estimation under the quasi-steady state operating condition, (2) is discretized as well, yielding the similar formula as (5) and (6) except for setting $\boldsymbol{x}_{k} \approx \boldsymbol{x}_{k-1}$. The use of conventional measurements from remote terminal units is sufficient. Conversely, when the system is operating under transient conditions, synchrophasor measurements may be the only source of information to carry out DSE. At the local level (e.g. generation station, substation or FACTS device site), digital recorders or protection devices [6], also referred to as intelligent electronic devices, can provide the required synchronized information to execute DSE, possibly including key parameters of the monitored device.

Remark: for the existing DSEs, not all the equality and the inequality constraints are duly considered, for example, the inequality constraints of the states, control inputs, and model parameters. The latter may cause serious concerns, such as algorithm divergence, violation of physical laws, obtaining only sub-optimal solutions, to name a few. Thus, DSE methods that take into account all constraints are needed.

\section{B. Dynamic State Estimation}

In order to estimate the dynamic state vector or model parameters of the general discrete-time state space model shown in (5)-(6), various nonlinear filters developed within the Kalman filter framework are used. It typically consists of two-steps [38], namely a prediction step using (5), and a filtering/update step using (6). Specifically, given the state estimate at time step $k-1, \widehat{\boldsymbol{x}}_{k-1 \mid k-1}$, with its covariance matrix, $\boldsymbol{P}_{k-1 \mid k-1}$, the predicted state vector is calculated from (5) directly or through a set of points drawn from the probability distribution of $\widehat{\boldsymbol{x}}_{k-1 \mid k-1}$, which is dependent on the assumed probability distributions of $\boldsymbol{w}_{k}$. As for the filtering step, the predictions are used together with the measurements at time step $k$ to estimate the state vector and its covariance matrix. Depending on how the state statistics are propagated, different Kalman filters are available, such as extended Kalman filter (EKF), unscented Kalman filter (UKF), ensemble Kalman filter (EnKF), particle filter (PF) [39], to name a few. They are reviewed and discussed in Section IV.

The derived state space equations (5)-(6) allow DSE to be applied rigorously to any power system, in any condition, provided the available computing power is sufficient for the size of the problem being addressed and the assumptions that all the equality and inequality constraints are appropriately enforced. The term 'dynamic', when applied to a quasi-steady 
state operating condition may be misleading, as the system dynamics are actually assumed to be absent/negligible and the state transitions are determined solely by the smooth evolution. In fact, under this scenario, we call the developed estimators as either FASE or TSE.

Remarks: The state vector can be the enlarged one that contains $\boldsymbol{x}$ and $\boldsymbol{y}$ simultaneously, or the reduced one ( $\boldsymbol{x}$ or $\boldsymbol{y}$ ), depending on whether algebraic or dynamic states are being estimated; the state vector $\boldsymbol{x}$ can be expanded to include uncertain parameters of generators and associated controllers. On the other hand, except for the widely used recursive estimators that are based on the Kalman filter framework, other non-recursive estimators can also be adopted for the state and parameter estimation, such as the infinite impulse response filter, the finite impulse response filter [40], to name a few.

\section{Forecasting-Aided State Estimation}

The so-called FASE is a particular application of DSE concept to quasi-steady state conditions, in which the statetransition model (change in operating point) is driven only by slow enough stochastic changes in the power injections (demand and generation) and the dynamics of $\boldsymbol{x}_{k}$ are sufficiently small to be neglected. In addition, unlike the original DSE formulation, in the FASE approach, the state-transition model (5) is assumed to be linear, leading to

$$
\begin{aligned}
\boldsymbol{y}_{k} & =\boldsymbol{A}_{k} \boldsymbol{y}_{k-1}+\boldsymbol{\zeta}_{k-1}+\boldsymbol{w}_{k}, \\
\boldsymbol{z}_{k} & =\boldsymbol{h}\left(\boldsymbol{y}_{k}, \boldsymbol{p}\right)+\boldsymbol{v}_{k}
\end{aligned}
$$

where $\boldsymbol{y}_{k}$ represents the algebraic state variables that specifically refer to the bus voltage magnitudes and angles; furthermore, instead of resorting to the nonlinear state-transition models, the required transition matrix $\boldsymbol{A}_{k}$ and the trend vector $\boldsymbol{\zeta}_{k-1}$, which is a function of $\boldsymbol{u}_{k}$, are identified from historical time series data. The widely used approaches for that are exponential smoothing regression or recursive least squares [12], [41]-[45]. In other words, the current values are computed from a weighted average of the most recent past values, with exponentially decreasing set of weights, disregarding any other information about the structure of the problem in hand.

When the input or the trend vector evolves smoothly, the FASE approach provides satisfactory results, generally better than the simpler TSE. However, potentially inaccurate results can be obtained in presence of sudden changes caused by loads, DERs, system topology, to name a few, as the state transition coefficients take a while to adapt to the new situation [45]. There are some mitigation approaches proposed in the literature to address that, such as the normalized innovation vector-based statistical test, the skewness test [42], [43], etc. However, these tests are generally under the Gaussian assumption, which is difficult to hold for true in practice, yielding unreliable detection thresholds. Furthermore, the differentiation among different anomalies remains a grand challenge. Further research work along this line is required. One of the advantages frequently associated with FASE is that the predicted state provides useful information for security analysis and preventive control functions. However, the benefits of such look-ahead or forecasting capability can be materialized in practice only if there is enough time for reaction, which may not be the case in transmission systems, featuring scanning rates of few seconds. The readers can refer to the two FASE review papers [46], [47] for more details.

\section{Tracking State Estimation}

With the assumptions that the state transition matrix $\boldsymbol{A}_{k}$ is an identity matrix and the change in the state vector is very small, the FASE simplifies to TSE [48]-[52]. Formally, the TSE model is expressed as

$$
\begin{aligned}
& \boldsymbol{y}_{k}=\boldsymbol{y}_{k-1}+\boldsymbol{w}_{k}, \\
& \boldsymbol{z}_{k}=\boldsymbol{h}\left(\boldsymbol{y}_{k}, \boldsymbol{p}\right)+\boldsymbol{v}_{k},
\end{aligned}
$$

where the change of state (random walk) $\boldsymbol{w}_{k}$ is assumed to be a white Gaussian noise with zero mean and known covariance matrix. One of the challenges in formulating TSE is the lack of an appropriate model to represent dynamics of the system state. TSE assumes a quasi-steady state state condition where the system state remains unchanged other than an additive Gaussian noise $\boldsymbol{w}_{k}$. With the increasing penetration of DERs and flexible loads, the trend $\zeta_{k-1}$ is no longer negligible and the change of state cannot be simply replaced by a white Gaussian noise [12], [52]. This scenario is further aggravated in the presence of changes in network topology and parameters owing to line or transformer switching, or switching of capacitor banks or shunt reactors. As a result, it becomes a challenge to adopt TSE for practical applications.

\section{E. Static State Estimation}

The SSE arises from a further simplification of TSE, in which the state transition information is fully ignored and only the nonlinear measurement function is maintained. As a result, SSE has no memory of the states at the previous time steps [53]. It is worth pointing out that SSE may perform better than TSE or FASE in the presence of sudden changes. However, it does not have the capability to track system dynamics as compared to DSE. Furthermore, unlike DSE, it requires that the state vector be observable solely with the latest set of available measurements. In fact, adding pseudo-measurements through state or measurement forecasting to SSE is somewhat equivalent to performing a FASE [45], [47].

It is worth pointing out that a recent variation of SSE is the linear state estimation (LSE) [54]-[56], which uses voltage and current phasors from PMUs, leading to a linear measurement function:

$$
\boldsymbol{z}_{k}=\boldsymbol{H} \boldsymbol{y}_{k}+\boldsymbol{v}_{k}
$$

where $\boldsymbol{H}$ is a constant matrix that consists of system transmission line parameters. LSE is able to track the online voltage magnitude and angle of each bus at the PMU reporting rate. However, it does not track the actual dynamics of the system, which are the dynamic states of machines, loads, etc.

\section{Tools And Methods For Implementation of DSE}

In what follows, we present a thorough yet not comprehensive review of the tools and methods for the implementation of DSE. The system observability concept is also discussed. After that, the focus is turned to DSE itself; the objective is to provide a summary of the different approaches, highlight their pros and cons, and discuss their implementation challenges. 


\section{A. Observability Analysis for DSE}

Observability is defined as the ability to uniquely determine the states of the system from available measurements. For the SSE problem, observability analysis can be carried out by topological or numerical methods [4] and it results in a binary outcome. By contrast, for DSE, one may refer to strongly or weakly observable systems [57]-[59]. Furthermore, due to the nonlinearity and time-dependent nature of the problem, observability results are also time dependent. The observability of a linear time-invariant dynamical system can be readily determined by whether or not its observability matrix has full rank. As for the nonlinear system in (5)-(6), one can perform a linearization around a given operating point and assess its local observability. While this approach may be advantageous from the computational standpoint, it will occasionally lead to incorrect results under highly nonlinear operating conditions. We therefore advocate the use of methods that do not perform any linearization. To date, two methods have been proposed and they are described next.

1) Method based on the Lie derivatives: A nonlinear dynamical system is said to be observable at a state $\boldsymbol{x}_{0}$ if the nonlinear observability matrix obtained by using Lie derivatives at $\boldsymbol{x}=\boldsymbol{x}_{0}$ has a full rank [60]. This can be explained as follows: consider the nonlinear system given by (5)-(6), the Lie derivative of $h$ with respect to $f$ is defined as

$$
L_{f} \boldsymbol{h}=\nabla \boldsymbol{h} \cdot \boldsymbol{f} .
$$

From the definition (10),

$$
L_{f}^{0} \boldsymbol{h}=\boldsymbol{h}, \quad L_{f}^{k} \boldsymbol{h}=\frac{\partial L_{f}^{k-1} \boldsymbol{h}}{\partial \boldsymbol{x}} \cdot \boldsymbol{f} .
$$

Now, define $\Omega$ as

$$
\boldsymbol{\Omega}=\left[\begin{array}{ccc}
L_{f}^{0}\left(\boldsymbol{h}_{1}\right) & \ldots & L_{f}^{0}\left(\boldsymbol{h}_{m}\right) \\
L_{f}^{1}\left(\boldsymbol{h}_{1}\right) & \ldots & L_{f}^{0}\left(\boldsymbol{h}_{m}\right) \\
\vdots & \ldots & \vdots \\
L_{f}^{n-1}\left(\boldsymbol{h}_{1}\right) & \ldots & L_{f}^{n-1}\left(\boldsymbol{h}_{m}\right)
\end{array}\right],
$$

and a gradient operator

$$
\boldsymbol{O}=d \boldsymbol{\Omega}=\left[\begin{array}{ccc}
d L_{f}^{0}\left(\boldsymbol{h}_{1}\right) & \ldots & d L_{f}^{0}\left(\boldsymbol{h}_{m}\right) \\
d L_{f}^{1}\left(\boldsymbol{h}_{1}\right) & \ldots & d L_{f}^{0}\left(\boldsymbol{h}_{m}\right) \\
\vdots & \ldots & \vdots \\
d L_{f}^{n-1}\left(\boldsymbol{h}_{1}\right) & \ldots & d L_{f}^{n-1}\left(\boldsymbol{h}_{m}\right)
\end{array}\right]
$$

The observability matrix $\boldsymbol{O}$ must have rank $n$ in order for the system to be observable, and $n$ is the dimension of the state vector $\boldsymbol{x}$.

In [61], this approach is used to determine the observability of several test systems in which the synchronous generators are represented by the classical model. Furthermore, the ratio between the smallest and the largest singular values of the observability matrix is used as a measure of the degree of observability. This work is further extended in [59], where the synchronous generators are represented by the two-axis model, including the model of the IEEE-Type 1 excitation system. It is worth pointing out that the computation of the Lie derivativebased observability matrix can be cumbersome even for smallscale power systems. The automatic differentiation approach
[61] can be used to reduce the burden of calculating the Lie derivatives of higher order.

2) Method based on the empirical observability Gramian: The empirical observability Gramian [62], [63] provides a way to analyze the state-output behavior of a nonlinear system. Different from analysis based on linearization, it is defined over an operating region of the original system model and reflects the observability of the full nonlinear dynamics in the given domain. One important property is that the empirical observability Gramian for a system with multiple outputs is the summation of the empirical Gramian computed for each of the outputs individually [57]. Therefore, the added observability after any sensor placement can be evaluated by calculating the empirical observability Gramian for each sensor. In [57] the observability of the system states is quantified by the determinant of the empirical observability Gramian. In [58], the level of unobservability of a nonlinear system is evaluated by the unobservability index approximated by the smallest eigenvalue of a Gramian matrix. In [64], different measures of the empirical observability Gramian, including the determinant, the trace, the minimum eigenvalue, and the condition number are compared. It is shown that when the observability is weak, the minimum eigenvalue and the condition number are better measures of the observability. By contrast, when the observability is strong, the determinant is a better measure.

\section{B. Implementations of DSE}

From a Bayesian perspective, the DSE problem consists of recursively calculating some degree of belief in the state vector $\boldsymbol{x}_{k}$ at time $k$, given the data up to time $k$. Thus, it is required to calculate the conditional probability density function (pdf), $p\left(\boldsymbol{x}_{k} \mid \boldsymbol{z}_{1: k}\right)$. The latter may be obtained recursively by the state prediction and updating steps. Suppose that $p\left(\boldsymbol{x}_{k-1} \mid \boldsymbol{z}_{1: k-1}\right)$ at time $k-1$ is available, that is, state estimate at time step $k-1, \widehat{\boldsymbol{x}}_{k-1 \mid k-1}$, with its covariance matrix, $\boldsymbol{P}_{k-1 \mid k-1}$ is given. Then, the prediction step involves applying (5) to obtain the a priori pdf of the state at time $k$ via

$$
p\left(\boldsymbol{x}_{k} \mid \boldsymbol{z}_{1: k-1}\right)=\int p\left(\boldsymbol{x}_{k} \mid \boldsymbol{x}_{k-1}\right) p\left(\boldsymbol{x}_{k-1} \mid \boldsymbol{z}_{1: k-1}\right) d \boldsymbol{x}_{k-1}
$$

The probabilistic model of the state evolution $p\left(\boldsymbol{x}_{k} \mid \boldsymbol{x}_{k-1}\right)$ is defined by the system equation given by (5) and the assumed probability distribution of $\boldsymbol{w}_{k}$. At time $k$, when the measurements/observations $\boldsymbol{z}_{k}$ are available, the update step can be performed via Bayes' rule expressed as

$$
p\left(\boldsymbol{x}_{k} \mid \boldsymbol{z}_{1: k}\right)=\frac{p\left(\boldsymbol{z}_{k} \mid \boldsymbol{x}_{k}\right) p\left(\boldsymbol{x}_{k} \mid \boldsymbol{z}_{1: k-1}\right)}{p\left(\boldsymbol{z}_{k} \mid \boldsymbol{z}_{1: k-1}\right)}
$$

where the normalizing constant given by $p\left(\boldsymbol{z}_{k} \mid \boldsymbol{z}_{1: k-1}\right)=$ $\int p\left(\boldsymbol{z}_{k} \mid \boldsymbol{x}_{k}\right) p\left(\boldsymbol{x}_{k} \mid \boldsymbol{z}_{1: k-1}\right) d \boldsymbol{x}_{k}$ depends on the likelihood function $p\left(\boldsymbol{z}_{k} \mid \boldsymbol{x}_{k}\right)$, which is defined by the observation model (6) and the assumed probability distribution of $\boldsymbol{v}_{k}$.

The recurrence relations (14) and (15) form the basis for the optimal Bayesian solution. However, this recursive propagation of the a posteriori density is only a conceptual solution in general; it cannot be determined analytically for the nonlinear dynamical system model [65]. It is in this sense that the EKF, UKF, EnKF, PF, and their variants only approximate 
the optimal Bayesian solution under the Gaussian noise assumption. In what follows, a thorough but not comprehensive review of these filters for power system DSE is presented.

1) Linearization-based Methods: If the predicted state vector $\widehat{\boldsymbol{x}}_{k \mid k-1}$ and its associated statistics $p\left(\boldsymbol{x}_{k} \mid \boldsymbol{z}_{1: k-1}\right)$ are calculated from (5) directly through the Taylor series expansion, (14)-(15) will reduce to the EKF variants. Pioneering work along this line is done by [5] with the assumption that the instrumentation system at that time can measure power injections and frequency at generator buses accurately enough and at high enough sampling rates. With the advancement of PMU technologies, these assumptions can be alleviated. To this end, [66] proposed and investigated the benefits of adopting PMU data for real-time state estimation using EKF while [67], [68] leveraged data from relays, PMUs, FDRs to monitor and control system transients at the substation level. In [69], an EKF algorithm is proposed using model decoupling technique to estimate states and parameters of a classical generator model. Following their work, Ghahremani and Kamwa [70] proposed a modified EKF-based DSE to cope with cases where the field voltage is not accessible to metering due to brushless excitation systems. This work was later extended with the development of a decentralized DSE while relaxing the assumption of a known mechanical torque [71]. The linearization-based methods work well if the nonlinearity of the dynamical system is not strong and the derivatives of nonlinear equations can be calculated. However, the system can be operated under stressed conditions, exhibiting strong nonlinearity. Furthermore, the derivatives can be challenging for complicated models and discrete switching events. To address these issues, derivative-free methods are preferred.

2) Derivative-free based Methods: If $\widehat{\boldsymbol{x}}_{k \mid k-1}$ and its associated statistics $p\left(\boldsymbol{x}_{k} \mid \boldsymbol{z}_{1: k-1}\right)$ are calculated through a set of points drawn from the probability distribution of $\widehat{\boldsymbol{x}}_{k-1 \mid k-1}$, the resulted methods are derivative-free. The main ideas of these methods are to use either the deterministic sampling techniques such as the unscented transform and its variants or the Monte Carlo based sampling technique, to choose a set of sample points, which have the same mean and covariance as the a priori state statistics [65], [72], [73]. These points are then propagated through the non-linear functions $f$ and $h$, yielding an estimation of the a posteriori state statistics by using the Kalman filter structure, i.e., the mean and covariance estimates. Consequently, no difficult and complex calculation of Jacobian matrices is required. Depending on the types of the chosen points, different methods are proposed, including the UKF and its variants using sigma points, EnKF, PF and their variants using ensembles or particles, which are Monte Carlo sampling techniques.

An UKF-based DSE using a fourth-order generator model is proposed in [74] to estimate the states of a single-machine infinite-bus power system. Along the same lines, a centralized UKF is developed in [75], [76] for a multi-machine system, while a decentralized strategy that does not require transmission of local signals is advocated in [77], significantly increasing the computational efficiency. The decentralized $\mathrm{UKF}$ is further extended to estimate fourth-order generator model dynamic state variables, the unknown field voltage and mechanical torque simultaneously [78]. It demonstrated improved performance with regard to the approach proposed in [71]. While in [79], the decentralized UKF is performed by incorporating internal angle in the dynamic model and by decoupling the estimation process into two stages with continuous updating of measurement-noise variances. To further improve the estimation accuracy of the decentralized UKF, the measurement correlations of the PMUs are considered [80]. As an alternative derivative-free method, EnKF uses Monte Carlo methods to estimate the error covariance matrix of the background error/noise, get an approximation to the filtered states, and produce an ensemble of initial conditions for the forecasting system. The latter is different from the UKF as sigma points need to be recalculated after the state filtering at each time instant. An EnKF method is firstly applied to track dynamic states of generators and its sensitivities to initial state errors, measurement noise and unknown faults are investigated in [81]. That approach is further extended in [82] to consider time-correlated mechanical input power from DERs. While in [83], a maximum likelihood ensemble filter is proposed to estimate state variables by optimizing a nonlinear cost function and leveraging low-dimensional ensemble space to calculate Hessian preconditioning of the cost function. Different from the EnKF, PF uses the particles generated through Monte Carlo sampling to propagate and approximate the state statistics through nonlinear functions. An extended PF is proposed to estimate the fourth-order synchronous machine states using the iterative sampling and inflation of particle dispersion [84]. In [85], [86], the PF is applied to estimate synchronous machine states with detailed models.

3) Enhanced Numerical and Statistical Robustness: The Kalman-type filters work well only under the validity of certain assumptions [38]. First, the system process and observation noise are assumed to have at each time instant zero means and known covariance matrices $\boldsymbol{Q}_{k}$ and $\boldsymbol{R}_{k}$, respectively. Secondly, they are assumed to follow a Gaussian distribution, at which the filter is optimal with minimum variances. Finally, the system model is assumed to be known with good accuracy. However, for most practical power systems, these assumptions do not hold true. Indeed, $\boldsymbol{Q}_{k}$ and $\boldsymbol{R}_{k}$ are difficult to obtain in practice; the process and observation noise follow non-Gaussian distributions as demonstrated in [37]; the functions $\boldsymbol{f}$ and $\boldsymbol{h}$ are approximate; for instance, they may not account for all the nonlinearities of the system; some model parameter values may be unknown or incorrect; and the received measurements may be strongly biased due to impulsive communication noise, or cyber attacks [87].

In [39], it is shown that the performances of EKF, UKF, EnKF and PF are greatly degraded in the presence of observation outliers due to their lack of robustness. To mitigate this issue, the normalized innovation vector-based test [77], [85] is usually used to detect observation outliers despite the vulnerability of this test to innovation outliers and non-Gaussian noise. In [88], a distributed two-stage robust UKF-based DSE using the least-absolute-value (LAV) estimator is presented to handle observation outliers in the PMU measurements. This approach has the similar issues as the normalized innovation vectorbased test. To this end, the generalized maximum-likelihood 
(GM) iterated EKF (GM-IEKF) [89], [90] is proposed to deal with the observation and innovation outliers. However, its statistical efficiency is relatively low in the presence of nonGaussian system process and measurement noise. To address that, the robust GM-UKF [27], [28] is proposed that is able to achieve a high statistical efficiency under a broad range of non-Gaussian noise while maintaining the robustness to observation and innovation outliers. A reformulation of the GM-UKF with multiple hypothesis testing [29] further enables it to handle three types of outliers. Note that the three types of gross errors or outliers include observation, innovation, and structural outliers [28]. They can be caused by either an unreliable dynamical model or real-time synchrophasor measurements with data quality issues. However, these GMtype DSE may yield biased state estimates in the presence of large system uncertainties. To this end, the H-infinity EKF and UKF that rely on robust control theory are proposed [91], [92]. It is shown that H-infinity filter can bound the system uncertainties but lacks robustness to outliers and non-Gaussian noise.

Remark: every approach has its domain of feasibility and beyond that, its performance is no longer guaranteed. Due to numerical issues, model errors and cyber-attacks, to cite a few, the DSE may not converge. In this scenario, only the forecasted states from the dynamical system model are available. Since the system dynamic states cannot change immediately following a disturbance, the forecasted states are reasonably close to the true dynamic states given that the system dynamic model is accurate. Then, this type of information can still be useful for operation and control. If that is not the case, we may only use the state estimates at the previous time instant for further processing. This is similar to the traditional SSE. Future research about enhancing the robustness of DSE against divergence is required. DSE results should also be validated carefully to avoid converging to wrong state estimates. The data-driven methods or machinelearning-based tools can be taken as a complementary to help that. This is another direction for research.

\section{Parameter Estimation and Calibration using DSE}

The application of Kalman filter and its variants for the state and parameter estimation has been widely used in signal processing, communication, among others. Along this line, there are typically two ways for implementations [93]: i) joint estimation, that is, the unknown parameters are augmented with the system state variables for joint estimation. This joint state is estimated through a single Kalman filter recursion; ii) dual approach: it contains two parallel filters, one on the state and the other on the parameters; in other words, the parameters are treated as known within the state filter at any given time, $k$, while the states are treated as known in the parallel parameter filter. Note that these two approaches show little differences in the estimation results for most applications [93]. On the other hand, the observability analysis must be performed and the number of measurements should be sufficient to initiate parameter estimation. In the power system parameter estimation context, only the joint estimation is used. For example, the transient reactance and inertia of the classical generator are augmented with state variables for joint estimation [94]. In [95]-[97], the UKF, the square-root UKF and constrained iterated UKF are used to estimate highorder generator model state and parameters simultaneously. The UKF is also used to estimate dynamic load parameters [98] and the frequency and fundamental power components [99]. Due to observability issues, not all unknown parameters can be estimated. Therefore, sensitivity analysis is carried out to narrow down the number of candidate parameters for calibration. This strategy is widely used in the model validation and calibration, such as [26], [100], [101].

Remark: the implementation of DSE for large-scale systems is challenging. To deal with that, the distributed or decentralized implementations proposed in the literature are encouraged. Another way of achieving that goal is through the high-performance computing and parallel computing techniques [102], [103], which require further investigations. Furthermore, when augmenting the identified incorrect parameters into the state vector for calibration, local observability analysis should be carefully carried out to avoid multiple solutions as well as numerical issues.

\section{Centralized vs. Decentralized DSE}

There are two ways of DSE implementations, namely the centralized and decentralized ones. Centralized DSE assumes that the system is observable by PMUs and Kron reduction can be carried out to reduce the system to the generators' terminals. In addition, it requires accurate knowledge of each system component parameters as well as the real-time wide-area PMU measurements. By contrast, the decentralized DSE is implemented using only local PMU measurements. It assumes that the terminal bus of the interested dynamic components is observed by PMU measurements and local observability of dynamic states is satisfied. If a generator terminal bus is not equipped with PMU, a PMU-based linear state estimator [54]-[56] for that local system should be performed first. Then, the estimated measurements at the interested terminal buses can be obtained and the decentralized DSE is executed. Note that the local phasor data concentrator (PDC) is in charge of communicating and processing the PMU measurements. Centralized DSE allows us to achieve global monitoring and control applications and has good robustness to data quality and security issues as the measurement redundancy is high. However, it has large computational burden and strong assumptions about the accuracy of the whole dynamic system models. By contrast, decentralized DSE only needs local measurements at the terminal buses and the dynamic model of interested components, which is fast to execute and not impacted by the model inaccuracy of other system components. However, the local measurement redundancy is low and therefore decentralized DSE has difficulties in dealing with PMU data quality and security issues. On the other hand, with a decentralized DSE, only local controls are implemented. If coordinated control is deployed between different local DSEs, additional communication bandwidth is required, making the comparison with the communication cost of the centralized DSE difficult to assess. Choosing between the two implementation schemes depends on the applications 
and the communication infrastructures being used. It should be noted that for both centralized and decentralized DSE-based applications, time tags of the estimated dynamic state variables are required when the communication network is involved [104].

\section{CONCLUSIONS AND Future Work}

This paper summarizes the technical activities of the task force members during the first year. It discusses the motivations, concepts and key tools and methodologies that are used for dynamic state and parameter estimation. DSE is presented in a unified framework which uses commonly accepted notation and formulation in power system dynamics and control literature with the aim of establishing a firm baseline for future research and development efforts.

Although significant research work has been done for DSE, there is still room for improvement. Here, several important aspects listed below are recommended for future work:

- Enhancement of the accuracy and security of DSE: the quality of the filtered/updated state vector relies heavily on the quality of the measurements and the predicted states. Therefore, to obtain reliable state estimates for dynamic systems, robust state prediction and state filtering with good efficiency are required. From the statistical robustness point of view, a robust DSE should be designed to be robust against several data quality issues, such as non-Gaussian measurement noise, bad data, cyber attacks, missing data, etc. From the numerical robustness aspect, a robust DSE should be developed, such as robust squareroot type filters or other alternatives that can converge under various operation conditions. Also, as the system is always subject to various uncertainties, a robust DSE should be designed that can bound the influence of these uncertainties. Furthermore, hybrid approaches that leverage the strengths of different theories are more likely to be usable for complicated power systems. Finally, datadriven DSE based on artificial neural networks [105], [106] and operator theory [107] that do not rely on the power system model have been studied. Preliminary results indicate that these approaches can estimate the generators' rotor angle and/or rotor speed. More work along this line is needed to include the estimation of other dynamic state variables, and to validate them with real PMU data;

- Observability Analysis: the use of the smallest singular value of the observability matrix, be it the one obtained via Lie derivatives or other linear approximation approaches, for quantifying the degree of observability of a nonlinear dynamic system has not been formally proven. Although a large number of simulation cases has strongly confirmed its validity, more work is needed to develop a computationally efficient and theoretically proven implementation of observability analysis. It is also worthwhile investigating the relationship between linearized (small-signal approximation) and Lie derivativebased observability matrices in order to define the region of validity for the computationally attractive small-signal approximation, in particular during system transients;
- Dynamic Model Parameter Estimation and Anomaly Detection: the current DSE-based parameter estimation techniques mainly focus on synchronous generators. They can be extended for model validation and calibration of various system components, such as inverter-based renewable generation units, dynamic loads, etc. It is worth pointing out that if there exist gross model parameters, the parameter error detection and identification should be carried out first. Although this task is challenging for complicated models with many parameters, it is the key for parameter calibration to enhance the reliability and accuracy of system models. On the other hand, the DSE can be extended to detect the failures of various control devices by setting up appropriate criteria, including exciters, PSS, transformers, etc;

- Dynamic Security Assessment: it requires a robust and computationally efficient DSE in order to accurately and promptly initialize transient simulations. More work is needed to identify the major drawbacks and bottlenecks experienced by the industry in executing their current dynamic security assessment procedures and addressing them by robust DSE. These issues will motivate further research towards development of DSE, which may be routinely executed along with SSE in future EMS;

- Wide-Area System Integrity Protection Systems: enhanced system observability through internal angle and speed estimates will allow us to compute more accurate transient energy function and the latter may lead to several breakthroughs, such as faster out-of-step detection, more realistic location of runaway generator and minimal amount of generation/load to be shed in order to preserve system integrity without knowing the topology accurately;

- DSE of Renewable and Other Asynchronous Distributed Sources: wind, solar and other distributed sources of energy are playing an increasingly significant role in power generation. But, being asynchronous, intermittent and 'inertia-less', these sources are difficult to be controlled for global system stability. Developing DSE for these sources at their point of common coupling with the grid is a viable solution, using which we can accurately estimate the latent 'synthetic-inertia' in these sources, and use it for contributing to the rotor-angle stability of the system. Local DSE at each wind-turbine or photovoltaic panel, within a wind-farm or solar-park, can also be used for optimizing the performance of the whole farm or park.

\section{ACKNOWLEDGEMENT}

The authors would like to thank all IEEE TF members for their valuable discussions and suggestions during web and face-to-face meetings. We also thank the anonymous reviewers for helping us to improve the quality of the paper.

\section{REFERENCES}

[1] M. Ilić and S. Liu, Hierarchical Power Systems Control: Its Value in a Changing Industry. Springer, 1996.

[2] P. Kundur, et al., "Definition and classification of power system stability IEEE/CIGRE joint task force on stability terms and definitions," IEEE Trans. Power Syst., vol. 19, no. 3, pp. 1387-1401, 2004.

[3] F. C. Schweppe, J. Wildes, "Power system static state estimation-Part I, II, III," IEEE Trans. Power App. Syst., vol.89, no.1, pp.120-135, 1970. 
[4] A. Abur and A. G. Expósito, Power System State Estimation: Theory and Application. Marcel Dekker, Inc., 2004.

[5] H. Modir, Robert A. Schlueter, "A dynamic state estimator for dynamic security assessment," IEEE Trans. Power Syst., vol. 100, no. 11, pp. 4644-4652, 1981.

[6] A. P. Meliopoulos, et al., "Dynamic state estimation based protection: status and promise," IEEE Trans. Power Delivery, vol. 32, no. 1, pp. 320-330, 2017.

[7] Y. Liu, A. P. Sakis Meliopoulos, et. al, "Dynamic state estimation based protection on series compensated transmission lines," IEEE Trans. Power Delivery, vol. 32, no. 5, pp. 2199-2209, 2017.

[8] R. Fan, et al. "Dynamic state estimation-based protection of power transformers," IEEE Power and Energy Society General Meeting, pp. $1-5,2015$.

[9] Y. Cui, R. G Kavasseri, S. M Brahma, "Dynamic state estimation assisted out-of-step detection for generators using angular difference," IEEE Trans. Power Delivery, vol. 32, no. 3, pp. 1441-1449, 2017.

[10] J. De La Ree, V. Centeno, J. Thorp, A. Phadke, "Synchronized phasor measurement applications in power systems," IEEE Trans. Smart Grid, vol. 1, no. 1, pp. 20-27, 2010.

[11] V. Terzija, et al., "Wide area monitoring, protection and control of future electric power networks," Proceedings of IEEE, vol. 99, no. 1, pp. 80-93, 2011.

[12] M. Hassanzadeh, C. Y. Evrenosoglu, L. Mili, "A short-term nodal voltage phasor forecasting method using temporal and spatial correlation," IEEE Trans. Power Syst., vol. 31, no. 5, pp. 3881-3890, 2016.

[13] I. Kamwa, et al., "Synchrophasors data analytics framework for power grid control and dynamic stability monitoring," IET Eng. Technol. Ref, pp. 1-22, 2016.

[14] J. Sanchez-Gasca, et al., "Identification of electromechanical modes in power systems," IEEE Task Force Rep., Jun. 2012.

[15] Y. Chen, M. Rice, K. Glaesemann, Z. Huang, "Sub-second state estimation implementation and its evaluation with real data," IEEE Power and Energy Society General Meeting, July 2015, pp. 1-5.

[16] S. Jin, Z. Huang, R. Diao, D. Wu, Y. Chen, "Comparative implementation of high performance computing for power system dynamic simulations," IEEE Trans. Smart Grid, vol. 8, no. 3, pp. 1387-1395, 2017.

[17] A. K. Singh, B. C. Pal, "Stability analysis of networked control in smart grids," IEEE Trans. Smart Grid, vol. 6, no. 1, pp. 381-390, 2015.

[18] M. Ghandhari, et al., "Control Lyapunov functions for controllable series devices," IEEE Trans. Power Syst., vol. 16, no. 4, pp. 689-694, 2001.

[19] A. K. Singh, B. C. Pal, "Decentralized nonlinear control for power systems using normal forms and detailed models," IEEE Trans. Power Syst., vol. 33, no. 2, pp. 1160-1172, 2018.

[20] A. K. Singh, B. C Pal, "Decentralized control of oscillatory dynamics in power systems using an extended LQR," IEEE Trans. Power Syst., vol. 31, no. 3, pp. 1715-1728, 2016.

[21] M. Ariff, B. C. Pal, "Adaptive protection and control in the power system for wide-area blackout prevention," IEEE Trans. Power Delivery, vol. 31, no. 4, pp. 1815-1825, 2016.

[22] H. Albinali, A. P. Meliopoulos, "A centralized substation protection scheme that detects hidden failures," in Proc. IEEE Power Energy Soc. Gen. Meeting, Boston, MA, USA, 2016, pp. 1-5.

[23] E. Farantatos, R. Huang, George J. Cokkinides, A. P. Meliopoulos, "A predictive generator out-of-step protection and transient stability monitoring scheme enabled by a distributed dynamic state estimator," IEEE Trans. Power Delivery, vol. 31, no. 4, pp. 1826-1835, 2016.

[24] "Next generation online dynamic security assessment," Final report, Power Systems Engineering Research Center, 2011.

[25] "Power plant dynamic model verification using PMUs," Reliability Guideline, NERC, 2016.

[26] Z. Huang, et al., "Generator dynamic model validation and parameter calibration using phasor measurements at the point of connection," IEEE Trans. Power Syst., vol. 28, no. 2, pp. 1939-1949, 2013.

[27] J. B. Zhao, L. Mili, "Robust unscented Kalman filter for power system dynamic state estimation with unknown noise statistics," IEEE Trans. Smart Grid, 2017.

[28] J. B. Zhao, L. Mili, "A robust generalized-maximum likelihood unscented Kalman filter for power system dynamic state estimation," IEEE Journal of Selected Topics in Signal Processing, 2018.

[29] J. B. Zhao, L. Mili, "Power system robust decentralized dynamic state estimation based on multiple hypothesis testing," IEEE Trans. Power Syst., vol. 33, no. 4, pp. 4553-4562, 2018.

[30] J. Chow. Power system coherency and model reduction. New York: Springer, 2013.
[31] J. B. Zhao, L. Mili, F. Milano, "Robust frequency divider for power system online monitoring and controls," IEEE Trans. Power Syst., vol. 33, no. 4, pp. 4414-4423, 2018.

[32] J. B. Zhao, Y. Tang, V. Terzija, "Robust online estimation of power system center of inertia frequency," IEEE Trans. Power Syst., vol. 34, no. 1, pp. 821-825, 2019.

[33] A. Rouhani, A. Abur, "A robust dynamic state estimator against exciter failures," North American Power Symposium, pp. 1-6, 2016.

[34] G. Anagnostou, et al., "Observer-based anomaly detection of synchronous generators for power systems monitoring," IEEE Trans. Power Syst., vol. 33, no. 4, pp. 4228-4237, 2018.

[35] P. Sauer, M. A. Pai. Power system dynamics and stability. Urbana, 1998.

[36] M. Crow. Computational methods for electric power systems. CRC Press, 2002

[37] S. Wang, et al., "Assessing Gaussian assumption of PMU measurement error using field data," IEEE Trans. Power Delivery, 2017.

[38] D. Simon. Optimal State Estimation. Hoboken, Wiley, 2006.

[39] N. Zhou, et al., "Dynamic state estimation of a synchronous machine using PMU data: A comparative study," IEEE Trans. Smart Grid., vol. 6, no. 1, pp: 450-460, 2015.

[40] J. Proakis, D. Manolakis, Digital Signal Processing: Principles, Algorithms, and Applications, Prentice-Hall, 1996.

[41] A. Da Silva, M. Do Coutto Filho, J. De Queiroz, "State forecasting in electric power systems," IEE Proceedings-Generation, Transmission and Distribution, vol. 130, no. 5, pp. 237-244, 1983.

[42] A. Da Silva, M. Do Coutto Filho, J. Cantera, "An efficient dynamic state estimation algorithm including bad data processing," IEEE Trans. Power Syst., vol. 2, no. 4, pp. 1050-1058, 1987.

[43] P. Rousseaux, D. Mallieu, T. Van Cutsem, M. Ribbens-Pavella, "Dynamic state prediction and hierarchical filtering for power system state estimation," Automatica, vol. 24, no. 5, pp. 595-618, 1988.

[44] K. Shih, S. Huang, "Application of a robust algorithm for dynamic state estimation of a power system," IEEE Trans. Power Syst., vol. 17, no. 1, pp. 141-147, 2002.

[45] J. B. Zhao, et al., "Robust forecasting aided power system state estimation considering state correlations", IEEE Trans. Smart Grid, vol. 9, no. 4, pp. 2658-2666, 2018.

[46] N. Shivakumar, A. Jain, "A review of power system dynamic state estimation techniques," IEEE Power System Technology Conference, pp. 1-6, 2008

[47] M. B. Do Coutto Filho, J. C. S. de Souza, "Forecasting-aided state estimation-Part I: Panorama," IEEE Trans. Power Syst., vol. 24, no. 4, pp. 1667-1677, 2009.

[48] A. Debs, R. E. Larson, "A dynamic estimator for tracking the state of a power system," IEEE Trans. Power App. Syst., vol. PAS 89, no.7, pp. 1670-1678, 1970.

[49] K. Nishiya, J. Hasegawa, T. Koike, "Dynamic state estimation including anomaly detection and identification for power systems," IEE Proceedings-Generation, Transmission and Distribution, vol. 129, no. 5, pp. 192-198, 1982.

[50] N. G. Bretas, "An iterative dynamic state estimation and bad data processing," International Journal of Electrical Power and Energy Systems, vol. 11, no. 1, pp. 70-74, 1989.

[51] A. Bahgat, M. Sakr, A. El-Shafei, "Two level dynamic state estimator for electric power systems based on nonlinear transformation," IEE Proceedings-Generation, Transmission and Distribution, vol. 136, no. 1, pp. 15-23, 1989

[52] X. Bian, et al., "Joint estimation of state and parameter with synchrophasors-Part I: state tracking," IEEE Trans. Power Syst., vol. 26, no. 3, pp. 1196-1208, 2011.

[53] F. C. Schweppe and D. Rom, "Power system static state estimation, Part II: Approximate model," IEEE Trans. Power App. Syst., vol. PAS-89, no. 1 , pp. 125-130, 1970.

[54] M. Gol, A. Abur, "LAV based robust state estimation for systems measured by PMUs," IEEE Trans. Smart Grid, vol. 5, no. 4, pp. 18081814, 2014. Apr. 2014.

[55] L. Zhang, A. Bose, A. Jampala, V. Madani, J. Giri, "Design, testing, and implementation of a linear state estimator in a real power system," IEEE Trans. Smart Grid, vol. 8, no. 4, pp. 1782-1789, 2017.

[56] C. Xu, A Abur, "A fast and robust linear state estimator for very large scale interconnected power grids," IEEE Trans. Smart Grid, vol. 9, no. 5, pp. 4975-4982, 2018.

[57] J. Qi, K. Sun, and W. Kang, "Optimal PMU placement for power system dynamic state estimation by using empirical observability gramian," IEEE Trans. Power Syst., vol. 30, no. 4, pp. 2041-2054, 2015. 
[58] K. Sun, J. Qi, and W. Kang, "Power system observability and dynamic state estimation for stability monitoring using synchrophasor measurements," Control Engineering Practice, vol. 53, pp. 160-172, 2016.

[59] A. Rouhani and A. Abur, "Observability analysis for dynamic state estimation of synchronous machines," IEEE Trans. Power Syst., vol. 32, no. 4, pp. 3168-3175, 2017.

[60] S. Diop, M. Filess, "On nonlinear observability," in Proc. ECC, Paris, France, pp. 152-157, 1991.

[61] G. Wang, et al., "Observability of nonlinear power system dynamics using synchrophasor data," Int. Trans. Electr. Energ. Syst., vol. 26, pp. 952-967, 2016.

[62] S. Lall, J. E. Marsden, and S. Glavaški, "Empirical model reduction of controlled nonlinear systems," 14th IFAC World Congress, Beijing China, 1999.

[63] S. Lall, J. E. Marsden, and S. Glavaški. "A subspace approach to balanced truncation for model reduction of nonlinear control systems," International Journal of Robust and Nonlinear Control, vol. 12, pp. 519-535, 2002.

[64] J. Qi, K. Sun, W. Kang, "Adaptive optimal PMU placement based on empirical observability gramian," in 10th IFAC Symposium on Nonlinear Control Systems, vol. 49, no. 18, pp. 482-487, 2016.

[65] M. S. Arulampalam, et al., "A tutorial on particle filters for online nonlinear/non-Gaussian Bayesian tracking," IEEE Trans. Signal Processing, vol. 50, no. 2, pp. 174-188, 2002.

[66] Z. Huang, K. Schneider, J. Nieplocha, "Feasibility studies of applying Kalman filter techniques to power system dynamic state estimation," in Proc. 8th Int. Power Eng. Conf., Singapore, pp. 376-382, 2007.

[67] E. Farantatos, et al., "PMU-based dynamic state estimation for electric power systems," in Proc. IEEE Power Eng. Soc. General Meeting, 2009, pp. $1-8$.

[68] S. Meliopoulos, et al., "Wide area dynamic monitoring and stability controls," in Proceedings of Bulk Power System Dynamic and Control, pp. 1-6, 2010

[69] L. Fan, Y. Wehbe, "Extended Kalman filtering based real-time dynamic state and parameter estimation using PMU data," Elect. Power Syst. Res. vol. 103, pp. 168-177, 2013.

[70] E. Ghahremani, I. Kamwa, "Dynamic state estimation in power system by applying the extended Kalman filter with unknown inputs to phasor measurements," IEEE Trans. Power Syst., vol. 26, no. 4, pp. 2556-2566, 2011.

[71] E. Ghahremani, I. Kamwa, "Local and wide-area PMU-based decentralized dynamic state estimation in multi-machine power systems," IEEE Trans. Power Syst., vol.31, no.1, pp. 547-562, 2016.

[72] S. Julier, J. K. Uhlmann, "Unscented filtering and nonlinear estimation," Proceedings of the IEEE, vol. 92, no. 3, pp. 401-422, 2004.

[73] G. Evensen, "The ensemble Kalman filter: theoretical formulation and practical implementation," Ocean Dynamics, vol. 53, pp. 343-367, 2003.

[74] E. Ghahremani, I. Kamwa, "Online state estimation of a synchronous generator using unscented Kalman filter from phasor measurements units," IEEE Trans. Energy Convers., vol.26, no.4, pp.1099-1108, 2011.

[75] S. Wang, W. Gao, A. P. S. Meliopoulos, "An alternative method for power system dynamic state estimation based on unscented transform," IEEE Trans. Power Syst., vol. 27, no. 2, pp. 942-950, 2012.

[76] J. Qi, K. Sun, J. Wang, H. Liu, "Dynamic state estimation for multimachine power system by unscented Kalman filter with enhanced numerical stability," IEEE Trans. Smart Grid, vol. 9, no. 2, pp. 11841196, 2018.

[77] A. K. Singh, B. C. Pal, "Decentralized dynamic state estimation in power systems using unscented transformation," IEEE Trans. Power Syst., vol. 29, no. 2, pp. 794-804, 2014.

[78] G. Anagnostou, B. C. Pal, "Derivative-free Kalman filtering based approaches to dynamic state estimation for power systems with unknown inputs," IEEE Trans. Power Syst., vol. 33, no. 1, pp. 116-130, 2018.

[79] A. Singh, B. C. Pal, "Decentralized robust dynamic state estimation in power systems using instrument transformers," IEEE Trans. Signal Processing, vol. 66, no. 6, pp. 1541-1550, 2018.

[80] J. B. Zhao, "Power system dynamic state estimation considering measurement correlations," IEEE Trans. Energy Conversion, vol. 32, no. 4, pp. 1630-1632, 2017.

[81] Y. Liu, et al., "Application of ensemble Kalman filter in power system state tracking and sensitivity analysis," IEEE PES Transmission and Distribution conference, pp. 1-8, 2012.

[82] W. Rosenthal, et al., "Ensemble Kalman filter for dynamic state estimation of power grids stochastically driven by time-correlated mechanica input power," IEEE Trans. Power Syst., vol. 33, no. 4, pp. 3701-3710, 2018.
[83] B. Uzunoglu, M. AkifUlker, "Maximum likelihood ensemble filter state estimation for power systems," IEEE Trans. Instrumentation and Measurement, 2018.

[84] N. Zhou, et al., "Estimation of the dynamic states of synchronous machines using an extended particle filter," IEEE Trans. Power Syst., vol. 28, no. 4, pp. 4152-4161, 2013.

[85] K. Emami, et al., "Particle filter approach to dynamic state estimation of generators in power systems," IEEE Trans. Power Syst., vol. 30, no. 5, pp. 2665-2675, 2015.

[86] Y. Cui, R. Kavasseri, "A particle filter for dynamic state estimation in multi-machine systems with detailed models," IEEE Trans. Power Syst., vol. 30, no. 6, pp. 3377-3385, 2015.

[87] A. F. Taha, J. Qi, J. Wang, J. H. Panchal, "Risk mitigation for dynamic state estimation against cyber attacks and unknown inputs," IEEE Trans. Smart Grid, vol. 9, no. 2, pp. 886-899, 2018.

[88] A. Rouhani, A. Abur, "Linear phasor estimator assisted dynamic state estimation," IEEE Trans. Smart Grid, vol. 9, no. 1, pp. 211-219, 2018.

[89] J. B. Zhao, L. Mili, "Robust dynamic state estimator to outliers and cyber attacks," Proc. IEEE Power Energy General Meeting, 2017, pp. $1-5$.

[90] J. B. Zhao, M. Netto, L. Mili, "A robust iterated extended Kalman filter for power system dynamic state estimation," IEEE Trans. Power Syst., vol. 32, no. 3, pp. 2484-2486, 2017.

[91] J. B. Zhao, "Dynamic state estimation with model uncertainties using H-infinity extended Kalman filter," IEEE Trans. Power Syst., vol. 33, no. 1, pp. 1099-1100, 2018.

[92] J. B. Zhao, L. Mili, "A decentralized H-infinity unscented Kalman filter for dynamic state estimation against uncertainties," IEEE Trans. Smart Grid, 2018, in press.

[93] S. Haykin, Kalman Filtering and Neural Networks, John Wiley, Hoboken, 2001.

[94] M. Ariff, et al., "Estimating dynamic model parameters for adaptive protection and control in power system," IEEE Trans. Power Syst., vol. 30, no. 2, pp. 829-839, 2015.

[95] G. Valverde, et al., "Nonlinear estimation of synchronous machine parameters using operating data," IEEE Trans. Energy Conversion, vol. 26, no. 3, pp. 831-839, 2011.

[96] M. Huanga, W. Li, W. Yana, "Estimating parameters of synchronous generators using Square-root unscented Kalman filter," Electric Power System Research, vol. 80, pp. 1137-1144, 2010.

[97] A. Rouhani, A Abur, "Constrained iterated unscented Kalman filter for dynamic state and parameter estimation," IEEE Trans. Power Syst., vol. 33, no. 3, pp. 2404-2414, 2018.

[98] A. Rouhani, A Abur, "Real-time dynamic parameter estimation for an exponential dynamic load model," IEEE Trans. Smart Grid, vol. 7, no. 3, pp. 1530-1536, 2016.

[99] R. Regulski, V. Terzija, "Estimation of frequency and fundamental power components using an unscented Kalman filter," IEEE Trans. Instrumentation and Measurement, vol. 61, no. 4, pp. 952-962, 2012.

[100] Z. Huang, et al., "Application of extended Kalman filter techniques for dynamic model parameter calibration," in Proc. IEEE Power Energy Gen. Meet., 2009

[101] R. Huang, et al., "Calibrating parameters of power system stability models using advanced ensemble Kalman filter," IEEE Trans. Power Syst., vol. 33, no. 3, pp. 2895-2905, 2018.

[102] S. Jin, Z. Huang, R. Diao, D. Wu, Y. Chen, "Comparative implementation of high performance computing for power system dynamic simulations," IEEE Trans. Smart Grid, vol. 8, no. 3, pp. 1387-1395, 2017.

[103] K. P. Schneider, Z. Huang, B. Yang, M. Hauer, J. Nieplocha, "Dynamic state estimation utilizing high performance computing methods," in IEEE PES Power System Conference and Exposition, Seattle, WA, March 15-18, 2009.

[104] A. Paul, I. Kamwa, G. Joos, "Centralized dynamic state estimation using a federation of extended Kalman filters with intermittent PMU data from generator terminals," IEEE Trans. Power Syst., vol. 33, no. 6, pp. 6109-6119, 2018.

[105] I. Kamwa, et al., "Sensorless ANN-based speed estimation of synchronous generator: improved performance through physically motivated pre-filters," in Proceedings of IEEE Neural Network Conference, pp. 1710-1718, 2006.

[106] A. D. Angel, et al., "Estimation of rotor angle of synchronous machines using artificial neural networks and local PMU based quantities," Neurocomputing, vol. 70, pp. 2668-2678, 2007.

[107] M. Netto, L. Mili, "A robust data-driven Koopman Kalman filter for power systems dynamic state estimation," IEEE Trans. Power Syst., vol. 33, no. 6, pp. 7228-7237, 2018. 